\title{
Kerr black holes in horizon-generating form
}

\author{
Sean A. Hayward \\ Department of Science Education, Ewha Womans University, \\ Seodaemun-gu, Seoul 120-750, Korea \\ hayward@mm. ewha.ac.kr \\ (Dated: 28th January 2004)
}

\begin{abstract}
New coordinates are given which describe non-degenerate Kerr black holes in dual-null foliations based on the outer (or inner) horizons, generalizing the Kruskal form for Schwarzschild black holes. The construction involves an area radius for the transverse surfaces and a generalization of the Regge-Wheeler radial function, both functions of the original radial coordinate only.
\end{abstract}

PACS numbers: $04.70 . \mathrm{Bw}$

It is hard to overstate the importance of the Kerr solution either in the theoretical history of black holes or as a model for actual astrophysical black holes. The solution was discovered [1] before black holes were understood as such, but it soon became a key ingredient in the classical theory of black holes 2, 3, 4, 5]. Uniqueness theorems indicate that this small family of solutions, parametrized by just mass and angular momentum, constitute all stationary black holes in vacuum, and therefore serve as good models for most real black holes for most of the time, from supernova remnants to supermassive black holes mysteriously found in galactic cores. Dynamical black holes have a partial theory being currently developed [6, 7, 8, 9, 10, 11, 12, 13, 14, 15], for which an appropriate understanding of Kerr black holes will also be necessary.

The generic Kerr metric is much more complex than the Schwarzschild metric, to which it reduces in the nonrotating case, and indeed Kerr discovered it almost a half-century after Einstein formulated General Relativity [16], as compared to Schwarzschild's few weeks 17]. While it has deservedly been one of the most studied exact solutions, there appears to be a peculiar lack of natural coordinates generating the outer (or inner) horizons, as in the Kruskal extension [18] of the Schwarzschild solution, which was so crucial for understanding its nature as a black hole. (There is an earlier paper of Fronsdal 19] which acknowledges priority to unpublished work of Kruskal; the Kruskal paper was actually written by Wheeler 20[). Specifically, recall that a non-degenerate Kerr black hole has two asymptotically flat external regions and two outer Killing horizons, intersecting in a bifurcation surface or wormhole throat [21, 22]. Kruskal's coordinates (or rather their sum and difference) describe a dual-null foliation of the space-time, meaning two families of null hypersurfaces intersecting in a two-parameter family of transverse spatial surfaces, such that the horizons are two of the hypersurfaces. Such coordinates are naturally adapted to the horizons and radiation propagation, both into the black hole and out of its vicinity. This Letter reports generalizations to non-degenerate Kerr black holes.

The Kerr solution with mass $m$ and angular momentum $m a$ in Boyer-Lindquist 21] coordinates $(t, r, \theta, \phi)$ can be given by the inverse metric

$$
g^{-1}=\frac{1}{\Sigma}\left(\Delta \partial_{r}^{2}+\partial_{\theta}^{2}+\frac{\Delta-a^{2} \sin ^{2} \theta}{\Delta \sin ^{2} \theta} \partial_{\phi}^{2}-\frac{4 m a r}{\Delta} \partial_{\phi} \partial_{t}-\frac{\Pi}{\Delta} \partial_{t}^{2}\right)
$$

where $\partial_{x}$ are the coordinate derivative vectors, $\partial_{x}\left(d x^{\prime}\right)=\delta_{x x^{\prime}}$, symmetric tensor products are implied and

$$
\begin{aligned}
\Delta & =r^{2}+a^{2}-2 m r \\
\Sigma & =r^{2}+a^{2} \cos ^{2} \theta \\
\Pi & =\left(r^{2}+a^{2}\right)^{2}-\Delta a^{2} \sin ^{2} \theta .
\end{aligned}
$$

The inverse metric is crucial in this adventure, since one is seeking null coordinates $x^{ \pm}: g^{-1}\left(d x^{ \pm}, d x^{ \pm}\right)=0$. For $a<m$, the Killing horizons are located at $r=r_{ \pm}$, where $r_{ \pm}=m \pm \sqrt{m^{2}-a^{2}}$ are the roots of $\Delta(r)=0$. Here the outer horizons $r=r_{+}$will be studied in detail, with modifications for the inner horizons $r=r_{-}$mentioned below. The desired transformation may be given in two steps, firstly to coordinates $\left(t^{*}, r^{*}, \vartheta, \varphi\right)$ defined by

$$
\begin{aligned}
t^{*} & =t-a \sin \theta \\
r^{*} & =\int \frac{R^{2}}{\Delta} d r \\
\vartheta & =\theta \\
\varphi & =\phi-\Omega(t-a \sin \theta)
\end{aligned}
$$


where $\Omega=a / 2 m r_{+}$is the angular velocity of the horizon and

$$
R(r)=\left(\left(r^{2}+a^{2}\right)^{2}-a^{2} \Delta\right)^{1 / 4}=\left(r^{4}+a^{2} r^{2}+2 m a^{2} r\right)^{1 / 4} .
$$

Secondly one may transform to $\left(x^{+}, x^{-}, \vartheta, \varphi\right)$, where

$$
x^{ \pm}= \pm e^{\kappa\left(r^{*} \pm t^{*}\right)}
$$

and $\kappa$ is determined below to be the surface gravity. The last step constructs dual-null coordinates $x^{ \pm}$by a generalization of Kruskal's method, having first identified appropriate coordinates $\left(t^{*}, r^{*}\right)$. The colatitude $\vartheta$ has been renamed merely for clarity in partial derivatives, and the new longitude $\varphi$ can be partly understood by noting that it is normal to the horizon-generating Killing vector $\chi=\partial_{t}+\Omega \partial_{\phi}: \chi(d \varphi)=\chi(d \vartheta)=0$. The same is true for $\phi-\Omega t$ alone [23], but it ceases to be spatial at large radius. The key step is the identification of the new coordinate $t^{*}$, which absorbs mischievous $\theta$-dependent terms to leave the corresponding $r^{*}$ (such that $r^{*} \pm t^{*}$ are null) miraculously available as a function of $r$ alone. One may recognize $r^{*}$ as a generalization of the Regge-Wheeler 24] tortoise coordinate, named after the ancient paradox of Achilles and the tortoise, as $r=r_{+}$corresponds to $r^{*} \rightarrow-\infty$.

The coordinate derivative vectors are related by

$$
\begin{aligned}
\partial_{t} & =\kappa\left(x^{+} \partial_{+}-x^{-} \partial_{-}\right)-\Omega \partial_{\varphi} \\
\partial_{r} & =\frac{R^{2}}{\Delta} \kappa\left(x^{+} \partial_{+}+x^{-} \partial_{-}\right) \\
\partial_{\theta} & =\partial_{\vartheta}+\Omega a \cos \vartheta \partial_{\varphi}-\kappa a \cos \vartheta\left(x^{+} \partial_{+}-x^{-} \partial_{-}\right) \\
\partial_{\phi} & =\partial_{\varphi}
\end{aligned}
$$

where $\partial_{ \pm}=\partial_{x^{ \pm}}$. Here $r$ is retained as a function of $x^{+} x^{-}=-e^{2 \kappa r^{*}}$, determined implicitly by inverting (6) to give $r\left(r^{*}\right)$. By carefully arranged cancellations in $R^{4}+\Delta a^{2} \cos ^{2} \theta-\Pi=0$, the inverse metric transforms to

$$
g^{-1}=\frac{1}{\Sigma}\left(\frac{4 R^{4} \kappa^{2} x^{+} x^{-}}{\Delta} \partial_{+} \partial_{-}+2 \kappa\left(x^{+} \partial_{+}-x^{-} \partial_{-}\right)\left(\alpha \partial_{\varphi}-a \cos \vartheta \partial_{\vartheta}\right)+\partial_{\vartheta}^{2}+2 \Omega a \cos \vartheta \partial_{\vartheta} \partial_{\varphi}+\left(\frac{1}{\sin ^{2} \vartheta}-\beta\right) \partial_{\varphi}^{2}\right)
$$

where

$$
\begin{aligned}
& \alpha(r)=\frac{\Omega R^{4}-2 m a r}{\Delta} \\
& \beta(r)=\frac{\Omega^{2} R^{4}-4 \Omega \operatorname{mar}+a^{2}}{\Delta} .
\end{aligned}
$$

Note that $x^{ \pm}$are null by the absence of $\partial_{ \pm}^{2}$ components, and that $r=r_{+}$has been mapped to $x^{+} x^{-}=0$. Then $x^{ \pm}=0$ are the two branches of the outer horizons, with $x^{+}=x^{-}=0$ being the bifurcation surface. The Killing vector $\chi=\kappa\left(x^{+} \partial_{+}-x^{-} \partial_{-}\right)$has a saddle point at each angle of the bifurcation surface: it vanishes there, and generates the two branches of the horizons along the principal directions. Having constructed a dual-null foliation based on the horizons in particular coordinates, the remaining coordinate freedom consists of relabelling the null hypersurfaces, $x^{ \pm} \mapsto \hat{x}^{ \pm}\left(x^{ \pm}\right)$, and diffeomorphisms of a transverse surface, i.e. choice of angular coordinates.

The functions $\left(\alpha, \beta, x^{+} x^{-} / \Delta\right)$, each apparently singular at $\Delta=0$, are all actually regular at $r=r_{+}$. Firstly, since $\Delta=\left(r-r_{+}\right)\left(r-r_{-}\right)$, near $r=r_{+}$one has $\left.r^{*} \sim\left(R^{2} /\left(r-r_{-}\right)\right)\right|_{r=r_{+}} \ln \left(r-r_{+}\right)$, so $e^{2 \kappa r^{*}} \sim r-r_{+}$if

$$
\kappa=\left.\frac{r-r_{-}}{2 R^{2}}\right|_{r=r_{+}}=\frac{\sqrt{m^{2}-a^{2}}}{2 m r_{+}}
$$

which is the usual surface gravity. Then $x^{+} x^{-} / \Delta \sim-1 /\left(r-r_{-}\right)$is regular at $r=r_{+}$. Secondly one can rearrange

$$
\begin{aligned}
& \alpha=\Omega\left(\frac{\left(r+r_{+}\right)\left(r^{2}+a^{2}\right)}{r-r_{-}}+r_{+}^{2}\right) \\
& \beta=\Omega^{2}\left(\frac{\left(r+r_{+}\right)^{2}\left(r-r_{+}\right)}{r-r_{-}}+2 r_{+}^{2}+a^{2}\right)
\end{aligned}
$$

which are now manifestly regular at $r=r_{+}$. Then $g^{-1}$ is regular, except for the polar coordinate breakdown at $\sin \vartheta=0$. With this usual caveat, the coordinate chart covers the entire region outside the inner horizons. 
For the inner horizons, one can use the same formulae (5)-(17) if one replaces the angular velocity and surface gravity by $\Omega=a / 2 m r_{-}$and $\kappa=-\sqrt{m^{2}-a^{2}} / 2 m r_{-}$respectively. Thus it is convenient to use a negative surface gravity for the inner horizons, as is anyway natural for dynamical horizons [7, 8, 9, 10, 11, 12. Then the new coordinate chart covers the region inside the outer horizons, up to the discs $r=0$, where $R=0$. Apart from the ring singularities at $\Sigma=0$, the discs are regular and one can extend through to the internal asymptotically flat regions, which can also be covered by a similar chart. The ring singularities present topological obstructions to any encircling foliation by spheres.

The metric

$$
g=\frac{\Sigma}{\Delta} d r^{2}+\Sigma d \theta^{2}+\frac{1}{\Sigma}\left(\Pi \sin ^{2} \theta d \phi^{2}-4 m a r \sin ^{2} \theta d \phi d t-\left(\Delta-a^{2} \sin ^{2} \theta\right) d t^{2}\right)
$$

in the new coordinates may be found by inverting the coordinate transformation to give the coordinate differentials

$$
\begin{aligned}
d t & =d t^{*}+a \cos \vartheta d \vartheta \\
d r & =\frac{\Delta}{R^{2}} d r^{*} \\
d \theta & =d \vartheta \\
d \phi & =d \varphi+\Omega d t^{*} .
\end{aligned}
$$

An independent and more systematic method uses the general dual-null decomposition [25]

$$
g^{-1}=-2 e^{f} \partial_{+} \partial_{-}+2 e^{f}\left(s_{+} \partial_{-}+s_{-} \partial_{+}\right)-2 e^{f} s_{+} s_{-}+h^{-1}
$$

where $h^{-1}$ is the inverse metric of the (constant- $\left.x^{ \pm}\right)$transverse surfaces, $s_{ \pm}$are shift vectors and $f$ is a normalization function. In this case one finds

$$
\begin{aligned}
e^{f} & =-\frac{2 R^{4} \kappa^{2} x^{+} x^{-}}{\Delta \Sigma} \\
s_{ \pm} & = \pm \frac{\Delta}{2 R^{4} \kappa x^{ \pm}}\left(\alpha \partial_{\varphi}-a \cos \vartheta \partial_{\vartheta}\right) \\
h^{-1} & =\frac{1}{R^{4} \Sigma}\left(\Pi \partial_{\vartheta}^{2}+4 m a^{2} r \cos \vartheta \partial_{\vartheta} \partial_{\varphi}+\left(\frac{R^{4}}{\sin ^{2} \vartheta}-a^{2} r(r+2 m)\right) \partial_{\varphi}^{2}\right) .
\end{aligned}
$$

The determinant of $h^{-1}$ is just $1 / R^{4} \sin ^{2} \vartheta$, so it may be easily inverted to yield the transverse metric

$$
h=\frac{1}{\Sigma}\left(\left(r^{4}+r(r+2 m) a^{2} \cos ^{2} \vartheta\right) d \vartheta^{2}-4 m a^{2} r \cos \vartheta \sin ^{2} \vartheta d \vartheta d \varphi+\Pi \sin ^{2} \vartheta d \varphi^{2}\right)
$$

where symmetric tensor products are again implied. Then the metric is given by the general form [25]

$$
g=h+2 h\left(s^{+}\right) d x^{+}+2 h\left(s^{-}\right) d x^{-}+h\left(s_{+}, s_{+}\right)\left(d x^{+}\right)^{2}+h\left(s_{-}, s_{-}\right)\left(d x^{-}\right)^{2}+2\left(h\left(s_{+}, s_{-}\right)-e^{-f}\right) d x^{+} d x^{-}
$$

by calculating

$$
\begin{aligned}
h\left(s_{ \pm}\right) & = \pm \frac{\Delta}{2 \Sigma \kappa x^{ \pm}}\left(\left(\alpha+\Omega a^{2} \cos ^{2} \vartheta\right) \sin ^{2} \vartheta d \varphi-\left(1+\gamma \sin ^{2} \vartheta\right) a \cos \vartheta d \vartheta\right) \\
h\left(s_{ \pm}, s_{ \pm}\right) & =\frac{\Delta}{\Sigma\left(2 \kappa x^{ \pm}\right)^{2}}\left(\left(\beta+\Omega^{2} a^{2} \cos ^{2} \vartheta\right) \sin ^{2} \vartheta-1+\Sigma^{2} / R^{4}\right) \\
h\left(s_{+}, s_{-}\right) & =-\frac{\Delta}{4 \sum \kappa^{2} x^{+} x^{-}}\left(\left(\beta+\Omega^{2} a^{2} \cos ^{2} \vartheta\right) \sin ^{2} \vartheta-1+\Sigma^{2} / R^{4}\right)
\end{aligned}
$$

where

$$
\gamma(r)=\Omega \alpha-\beta=\frac{2 \Omega m a r-a^{2}}{\Delta}=\frac{2 \Omega m a}{r-r_{-}}
$$

The terms involving the shift vectors are quite complex as compared to those in the inverse metric. However, the transverse metric $h$ has an unexpectedly nice property: its area form is just $R^{2} \sin \vartheta d \vartheta \wedge d \varphi$. (All the usual coordinates 1, 21, 22, 23] have a transverse area form with more complex $\theta$-dependence). Thus the new radial function $R$ is revealed as the area radius of the transverse surfaces. This property is useful for evaluating surface integrals, most 
simply the area $4 \pi R^{2}$ of the transverse surfaces. Also, while the coordinates were intended only to cover the outer horizons, the angular coordinates $(\vartheta, \varphi)$ become standard spherical polar coordinates on round spheres as $R \rightarrow \infty$ : $h / R^{2} \rightarrow d \vartheta^{2}+\sin ^{2} \vartheta d \varphi^{2}$.

One might at first think that this dual-null foliation is unique, since the foliations of each horizon are generated by $\chi$ from the bifurcation surface. However, $\chi$ vanishes at the bifurcation surface, leaving the possibility to include it in different foliations of the horizons. In fact, another such foliation can be found simply by replacing $a$ with $-a$ in the definitions of $t^{*}$ (5) and $\varphi$ (8), which has the effect of changing the sign of $d \vartheta$ in the metric, or of $\partial_{\vartheta}$ in the inverse metric. The two sets of transverse surfaces have the same poles, but their equators are displaced relative to each other. The displacements are in opposite directions in the two external regions, so the two foliations are dual under left-right inversion of the space-time. The existence of such dual foliations is due to breaking the explicit symmetry $(t, \phi) \mapsto(-t,-\phi)$ in $\left(t^{*}, \varphi\right)$. One may choose whether to displace the time coordinate to the future or past in one universe, forcing the opposite choice to be made in the other universe. Despite this peculiarity, it seems likely that this pair of foliations is unique in some sense. For instance, one might conjecture that they are the only dual-null foliations based on the horizons for which the transverse area form is a function of $r$ times the area form of a unit sphere.

The new coordinates are so naturally adapted to Kerr horizons and radiation propagation that they are likely to have various applications, while the area radius $R$ and the generalized tortoise coordinate $r^{*}$ may themselves prove useful. Applications might include electromagnetic radiation powered by rotating black holes, gravitational radiation produced by perturbed black holes, quasi-normal modes, Cauchy-horizon instability and black-hole evaporation. It might also help in the search for practical definitions of energy-momentum and angular momentum in the absence of symmetries, instead using extrinsic properties of embedded surfaces. The author's main interest in this enterprise was to find the Kerr solution in a similar dual-null form to that which has proved useful in understanding dynamical black holes. Its role as a basic test case may now be employed to further develop a general theory of black-hole dynamics.

Supported by research grant "Black holes and gravitational waves" of Ewha Womans University.

[1] R P Kerr, Phys. Rev. Lett. 11, 237 (1963).

[2] J M Bardeen, B Carter \& S W Hawking, Comm. Math. Phys. 31, 161 (1973).

[3] S W Hawking \& G F R Ellis, The Large Scale Structure of Space-Time (Cambridge University Press 1973).

[4] C W Misner, K S Thorne \& J A Wheeler, Gravitation (Freeman 1973).

[5] R M Wald, General Relativity (University of Chicago Press 1984).

[6] S A Hayward, Phys. Rev. D49, 6467 (1994).

[7] S A Hayward, Class. Quantum Grav. 15, 3147 (1998).

[8] S A Hayward, Phys. Rev. Lett. 81, 4557 (1998).

[9] S A Hayward, Class. Quantum Grav. 17, 1749 (2000).

[10] S Mukohyama \& S A Hayward, Class. Quantum Grav. 17, 2153 (2000).

[11] S A Hayward, Class. Quantum Grav. 18, 5561 (2001).

[12] S A Hayward, Black holes: new horizons, in Proceedings of the Ninth Marcel Grossmann Meeting on General Relativity, ed. V G Gurzadyan, R T Jantzen \& R Ruffini (World Scientific 2002).

[13] A Ashtekar \& B Krishnan, Phys. Rev. Lett. 89, 261101 (2002).

[14] A Ashtekar \& B Krishnan, Phys. Rev. D68, 104030 (2003).

[15] I Booth \& S Fairhurst, Phys. Rev. Lett. 92, 011102 (2004).

[16] A Einstein, Preuss. Akad. Wiss. Berlin, Sitzber. 844 (1915).

[17] K Schwarzschild, Sitzber. Deut. Akad. Wiss. Berlin, Kl. Math.-Phys. Tech. 189 (1916).

[18] M D Kruskal, Phys. Rev. 119, 1743 (1960).

[19] C Fronsdal, Phys. Rev. 116, 778 (1959).

[20] J A Wheeler \& K Ford, Geons, Black Holes \& Quantum Foam: A Life in Physics (W W Norton 2000).

[21] R H Boyer \& R W Lindquist, J. Math. Phys. 8, 265 (1967).

[22] B Carter, Phys. Rev. 174, 1559 (1968).

[23] S Chandrasekhar, The Mathematical Theory of Black Holes (Oxford University Press 1992).

[24] T Regge \& J A Wheeler, Phys. Rev. 108, 1063 (1957).

[25] S A Hayward, Class. Quantum Grav. 10, 779 (1993). 